\title{
A Pilot Study of Risperidone, Olanzapine, and Haloperidol in Psychotic Youth: A Double-Blind, Randomized, 8-Week Trial
}

\author{
Linmarie Sikich*,', Robert M Hamer, ${ }^{1,2}$, Robert A Bashford', Brian B Sheitman ${ }^{1,3}$ and Jeffrey A Lieberman ${ }^{1,4}$ \\ 'Department of Psychiatry, School of Medicine, University of North Carolina at Chapel Hill, Chapel Hill, NC, USA; ${ }^{2}$ Department of Biostatistics, \\ School of Public Health, University of North Carolina at Chapel Hill, Chapel Hill, NC, USA; ${ }^{3}$ Dorothea Dix Hospital, Raleigh, NC, USA; \\ ${ }^{4}$ Department of Pharmacology, School of Medicine, University of North Carolina at Chapel Hill, NC, USA
}

\begin{abstract}
This pilot study was undertaken to estimate the acute antipsychotic effect size and side effect propensity of risperidone and olanzapine in the pediatric population, in comparison to haloperidol, a conventional antipsychotic with established efficacy. Risperidone and olanzapine are widely used as first-line treatments to ameliorate psychotic symptoms in youth, but their abilities to specifically treat children and adolescents presenting due to psychotic symptoms have not been rigorously studied. Subjects, selected because of prominent positive psychotic symptoms, were randomly assigned to double-blind, parallel treatment with risperidone, olanzapine, or haloperidol for 8 weeks. The primary outcome was reduction in the Brief Psychiatric Rating Scale for Children total score from baseline to termination. An exploratory, descriptive analysis was done to compare the three treatments. A total of 50 patients, 8-19 years, participated. All treatments reduced symptoms significantly with $p$-values (corrected for multiple comparisons) of 0.00 I 8 for each of the atypical agents and 0.012 for haloperidol. In all, $88 \%$ of subjects treated with olanzapine, $74 \%$ treated with risperidone, and $53 \%$ treated with haloperidol met response criteria. The primary side effects observed in all patients were mild to moderate sedation, extrapyramidal symptoms, and weight gain. Risperidone and olanzapine acutely reduced psychotic symptoms in this pediatric sample. Exploratory comparisons indicate the magnitude of the antipsychotic response with these atypical agents is comparable to that observed with haloperidol. However, youth treated with risperidone and olanzapine experienced weight gain and extrapyramidal effects that appear more prevalent and severe than reported in adults.
\end{abstract}

Neuropsychopharmacology (2004) 29, I33-145, advance online publication, 29 October 2003; doi: | 0. I 038/sj.npp. I 300327

Keywords: antipsychotic agents; risperidone; olanzapine; childhood schizophrenia; psychotic disorders; adolescent

\section{INTRODUCTION}

Child psychiatrists have embraced the use of atypical antipsychotics based largely on studies in adults with psychosis demonstrating reduced propensity of extrapyramidal side effects (EPS) including tardive dyskinesia and increased ability to reduce negative symptoms of the schizophrenia syndrome and case reports in the pediatric population (Lieberman, 1996; Kumra, 2000; Remschmidt et al, 2000; Toren et al, 1998). Indeed, the ratio of atypical antipsychotics to traditional antipsychotics used is greater among child $(2.7: 1)$ and adolescent $(3.8: 1)$ patients than adults $(1.6: 1)$ (PMATSLS2, 2002). In the pediatric population, antipsychotics are frequently used to treat symptoms

\footnotetext{
*Correspondence: L Sikich, Department of Psychiatry, Medical School Wing C, University of North Carolina at Chapel Hill, Room 245, CB \# 7I60, Chapel Hill, NC 27599-7I60, USA, Tel: + I 919966 8653; Fax: + I919966 8004, E-mail: Lsikich@med.unc.edu

Received 06 January 2003; revised 04 September 2003; accepted 08 September 2003

Online publication: 19 September 2003 at http://www.acnp.org/ citations/Npp09730303010/default.pdf
}

and behavioral problems other than psychosis. Over the past few years, randomized controlled studies of atypical agents have been conducted for the treatment of disruptive behaviors in autism spectrum disorders (Malone et al, 2001; McCracken et al, 2002) and conduct disorder in youth with low or subnormal intelligence (Findling et al, 2000; Aman et al, 2002; Snyder et al, 2002). Most of these studies have used relatively low doses of antipsychotic (eg risperidone mean of $1.75 \mathrm{mg}$, olanzapine mean of $7.9 \mathrm{mg}$ ).

Recent studies have also examined the adjunctive role of atypical antipsychotic treatment in youth with bipolar affective disorder. Kafantaris et al (2001a,b) examined 49 adolescents who had experienced psychotic symptoms as part of a manic episode, which resolved with combined lithium and antipsychotic treatment. All five subjects who openly discontinued the antipsychotic after a week of therapeutic lithium treatment (Kafantaris et al, 2001a) and $71 \%$ of the 42 subjects who openly discontinued antipsychotic after 4 weeks of therapeutic lithium treatment (Kafantaris et al, 2001b) experienced significant deterioration without the antipsychotic (haloperidol $5-10 \mathrm{mg} /$ day or risperidone up to $6 \mathrm{mg} /$ day). DelBello et al (2002) 
conducted a double-blind, placebo-controlled trial of adjunctive quetiapine (mean dose $432 \mathrm{mg} /$ day) in 30 adolescents with bipolar disorder, $47 \%$ of whom had psychotic symptoms. Youth treated with both quetiapine and valproate had greater reduction in manic symptoms over time than those treated with valproate alone. However, there was no difference between the groups in the extent of positive symptom reduction.

However, to date, there has been only one blinded study of an atypical antipsychotic specifically targeting youth with significant psychotic symptoms (Kumra et al, 1996). This study was limited to 21 patients with prepubertal onset of schizophrenia and refractory symptoms. Thus, it may have limited generalizability. Further, it examined the efficacy of clozapine, which is generally reserved as a second- or thirdline intervention due to its potential toxicities. This study found that clozapine had clear advantages over haloperidol with regard to efficacy in reducing both positive and negative symptoms. However, it also highlighted clozapine's potential for significant toxicity, which appears heightened in the pediatric population.

The limited number of rigorous antipsychotic studies focused on the treatment of psychosis in the pediatric population reflects the difficulty involved in conducting randomized, controlled trials in children with psychotic illnesses. The reasons for this are multiple, but poorly understood. Differential diagnosis of psychotic disorders in the pediatric population is frequently difficult due to the lack of longitudinal information about disease course. Indeed, some studies have found that as many as $55 \%$ of individuals initially presenting with psychotic symptoms are given different diagnoses upon follow-up evaluation 2-6 years later (Werry et al, 1991; McKenna et al, 1994). Many investigators and potential participants also have ethical concerns about withholding treatment by utilizing a placebo or limiting the use of concurrent antidepressants and mood stabilizers, which have become the community standard of care, given the significant risk of suicide and violence in this population. Finally, recruitment of a sufficiently large sample is frequently challenging. Recruitment may be limited as a result of the relatively low incidence of psychotic disorders in youth, the increased burden on participants created by study assessments, the discomfort associated with administering blinded medications to children, and the social stigma associated with participation in research. Together these problems frequently result in relatively small, diagnostically imprecise and heterogeneous samples that are treated with multiple medications rather than a single agent or placebo.

More rigorous studies of first-line atypical antipsychotics in children and adolescents with significant psychotic symptoms are needed to guide the clinical care in this highly vulnerable population. Study of a broad sample of children whose psychotic symptoms occur in multiple diagnostic contexts may increase the generalizability of results. In addition, it is important to determine the safety and tolerability of relatively high doses of the newer medications in the pediatric population, given the growing awareness of the increased risk of atypical antipsychoticassociated diabetes in adults (Henderson, 2002; Newcomer et al, 2002; Sernyak et al, 2002; Wirshing et al, 1998), case reports describing significant side effects when atypicals are used in children and adolescents (Selva and Scott, 2001; Buitelaar et al, 2001; Kelly et al, 1998; Martin et al, 2000; Ratzoni et al, 2002; Malone et al, 1999; Buitelaar and Willemsen-Swinkels, 2000; Wudarsky et al, 1999), welldocumented developmental differences in the side effect profiles of medications, and the likelihood that children with psychosis will remain on antipsychotics for years.

This study was developed to provide prospective, blinded information about the ability to reduce positive psychotic symptoms and safety of the two atypical antipsychotics that are most widely used in the pediatric population at this time - risperidone and olanzapine - in order to inform the design of larger, more rigorous trials as quickly as possible. Due to concerns about withholding treatment in psychotic youth, haloperidol was used as the comparator rather than placebo. We felt this was justified because there have been two placebo-controlled studies establishing haloperidol's efficacy in schizophrenic youth (Pool et al, 1976; Spencer and Campbell, 1994).

\section{METHODS}

\section{Participants}

Subjects were selected on the basis of having at least one positive psychotic symptom of moderate or greater severity on the Brief Psychiatric Rating Scale for Children (BPRS-C) (Overall and Pfefferbaum, 1982), which had been present throughout the past 2 weeks, and full scale IQ greater than 69. Permitted primary diagnoses were Psychosis NOS, Schizophreniform Disorder, Schizophrenia, Schizoaffective Disorder, Delusional Disorder, Major Depression with Psychotic Features, and Bipolar Affective Disorder with Psychotic Features. Diagnoses were based upon medical record review, detailed clinical examination by a child psychiatrist (LS), and a structured diagnostic interview administered by social workers or a psychiatric nurse specialist, all with extensive child psychiatry experience. All diagnosticians achieved 0.85 inter-rater reliability on the NYU form of The Schedule for Affective Disorders and Schizophrenia for School-Age Children (K-SADS-P) (Chambers et al, 1985) and on the Structured Clinical Interview for DSM-IV (SCID) (First et al, 1997). The K-SADS-P and the psychotic disorders section of the SCID were used for most subjects; the SCID alone was used for 18- and 19-year olds $(n=5)$. Diagnoses were re-evaluated at completion of the study.

Exclusion criteria were psychotic symptoms that appeared to result from acute substance intoxication or withdrawal, a history of serious adverse reactions or nonresponse to an adequate trial of any of the study medications during this psychotic episode, prior diagnosis of a pervasive developmental disorder, serious medical or neurological disorder, pregnancy or refusal to practice contraception, or imminent risk in current setting to harm self or others. Individuals with comorbid diagnoses of posttraumatic stress disorder (PTSD) were permitted only if the majority of psychotic symptoms appeared unrelated to the PTSD. Similarly, individuals with a current or recent diagnosis of attention deficit hyperactivity disorder (ADHD), Tourette's syndrome (TS), or obsessive compulsive disorder (OCD), or with a past history of substance 


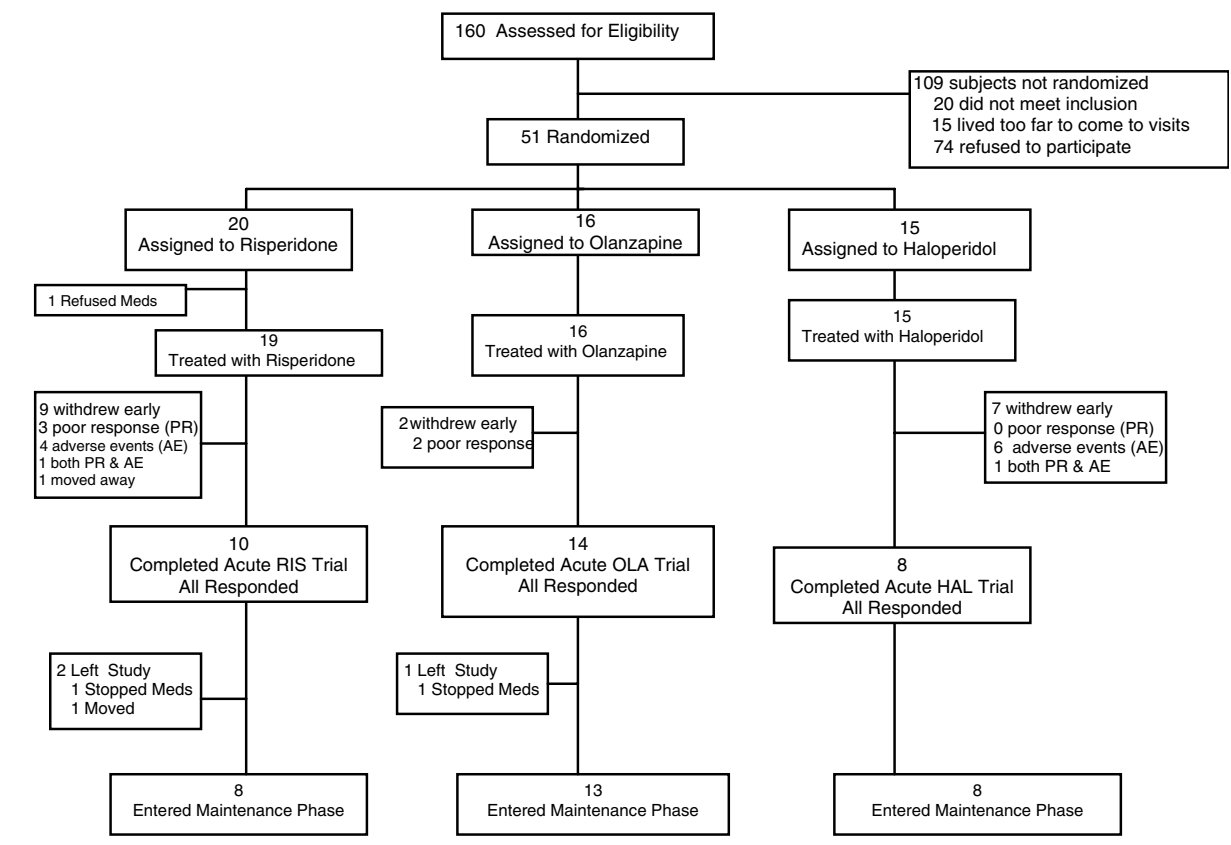

Figure I Subject flow.

abuse or dependence were allowed to participate only if their psychotic symptoms were not better accounted for by the comorbid disorder.

Participants were recruited from the in-patient and outpatient services of the UNC Healthcare system and Dorothea Dix Hospital as well as the practices of other psychiatrists throughout North Carolina. The intended sample size was 75 . However, recruitment difficulties resulted in a final sample size of 50 youth (see Figure 1). A sample of this size only has sufficient power to detect differences with large effect sizes, but not differences with moderate to small effect sizes. The protocol was approved by the Institutional Review Board of the University of North Carolina at Chapel Hill School of Medicine. Each child and his/her guardian gave assent and consent prior to entering the study. Their continued verbal assent and consent were obtained throughout the course of their involvement in the study.

\section{STUDY DESIGN}

Subjects were randomly assigned to double-blind, flexibledose, parallel treatment with risperidone, olanzapine, or haloperidol. The computer-generated randomization schedule was stratified by age (8-11 years or $\geqslant 12$ years) in order to ensure that younger children, who might have different metabolism or adverse reactions to the medications, were equally represented in all treatment groups. Further, subjects whose psychotic symptoms occurred either in the context of schizophrenia spectrum illnesses or affective disorders were included to provide data that more closely mirrors clinical practice. All subjects were randomly assigned to treatment without regard to diagnosis. A placebo lead-in was not used because of concerns about delaying treatment. The dose of medication was titrated to a moderate target dose (risperidone $-0.5-3 \mathrm{mg}$ in $0.5 \mathrm{mg}$ increments, olanzapine $-2.5-12.5 \mathrm{mg}$ in $2.5 \mathrm{mg}$ increments, and haloperidol- $1-5 \mathrm{mg}$ in $1 \mathrm{mg}$ increments) over 1-2 weeks. The rate of titration was determined by participant response. A slower titration was used if participants had significant side effects; doses were maintained below the target if subjects demonstrated marked improvement at a lower dose. If subjects continued to show significant psychotic symptoms after 2 weeks, the dose could be titrated upward to maximal doses of risperidone $6 \mathrm{mg}$ (in $1 \mathrm{mg}$ increments), olanzapine $20 \mathrm{mg}$ (in $2.5 \mathrm{mg}$ increments), or haloperidol $8 \mathrm{mg}$ (in $1 \mathrm{mg}$ increments). The antipsychotic dose could also be lowered if clinically indicated. Psychoeducation and supportive psychotherapy were provided to all subjects and their families during the course of the study. Subjects who were in-patients also received routine group, recreational, and occupational therapies.

The treatment period of this acute trial was 8 weeks. Subjects with intolerable side effects and/or persistent or worsening symptoms were withdrawn as clinically indicated. Visits occurred weekly. At the end of acute treatment, the response status of each subject (defined by a Clinical Global Impressions (Guy, 1976)-Improvement (CGI-I) score of ' 1 ' or ' 2 ' and at least a $20 \%$ reduction in the BPRS-C total score) was determined. Those who responded were eligible to continue double-blind treatment for an additional 12 weeks; the results of this maintenance trial are reported separately.

No subjects received prophylactic anticholinergic medications. Instead, at the first sign of clinically significant extrapyramidal symptoms (EPS), benztropine (titrated between 1 and $3 \mathrm{mg} /$ day) was provided, or, if the subject's clinical condition permitted, the antipsychotic was decreased. If the subject was not able to tolerate benztropine, trihexyphenidyl (4-6 mg/day) was used. If the subject was not able to tolerate either, amantadine $(200 \mathrm{mg} /$ day $)$ was used. Similarly, propranolol (20-60 mg/day) was 
provided for akathisia if antipsychotic dose reduction was not feasible. Lorazepam $(0.5-3 \mathrm{mg} /$ day $)$ was offered throughout the trial if needed to decrease agitation and anxiety.

Subjects taking an antipsychotic upon entry were tapered off that antipsychotic over 1-2 weeks beginning two days after the study medication was initiated. Antidepressants and mood stabilizers were permitted if the subject had been taking the agent for more than 4 weeks preceding entry without significant improvement in psychotic symptoms or if clinically significant affective symptoms persisted after 4 weeks of study treatment in order to meet the clinical needs of patients, provide greater generalizability, and enhance participation in the study. These thymoleptic medications could also be weaned after 4 weeks if clinically indicated. The antidepressants utilized were fluoxetine $(10-20 \mathrm{mg}$, two subjects (one after 4 weeks) in olanzapine group), sertraline (25-150 mg, two in risperidone group, one in olanzapine group, three in haloperidol group), paroxetine (20 mg, one in olanzapine group, one in haloperidol group), citalopram $(20 \mathrm{mg}$, one in risperidone group, one in haloperidol group), fluvoxamine (150 $\mathrm{mg}$, one in olanzapine group), clomipramine (100 $\mathrm{mg}$, one in risperidone group), buproprion (150-300 mg, two after 4 weeks in olanzapine group, one after 4 weeks in haloperidol group), venlaflaxine (150 mg, one in haloperidol group), and trazadone ( $100 \mathrm{mg}$, one in risperidone group). The mood stabilizers used were lithium (900-1200 mg, one in olanzapine group, one in haloperidol group), valproate (625-1000 mg, three in risperidone group, one in olanzapine group, two in haloperidol group), carbamazepine $(400-600 \mathrm{mg}$, one in haloperidol group), gabapentin (600-2400 mg, one in risperidone group, one in haloperidol group), and lamotrigine $(200 \mathrm{mg}$ in combination with gabapentin, one in risperidone group).

\section{EVALUATION OF EFFICACY}

The primary outcome measure for the within group analyses was the change in the BPRS-C total score from baseline to end point. The BPRS-C is a 21-item, clinicianrated, 0-6 scale assessing psychiatric symptoms frequently seen in children receiving psychiatric treatment. The total score has been shown to be sensitive to treatment effects in both depressive illness and schizophrenia (Spencer and Campbell, 1994; Emslie et al, 1997; Hughes et al, 2001). Further, the total score more comprehensively assesses the range of behavioral problems observed in youth with psychosis than the thinking disturbance and withdrawal subscales do (McClellan et al, 2002). It was supplemented with the disorganized thinking item from the adult Brief Psychiatric Rating Scale (Overall and Gorham, 1962), which has not been specifically validated in the pediatric population.

Secondary outcome measures included the Clinical Global Impressions-Severity score (CGI-S), the CGI-I, and the total score of the Children's Psychiatric Rating Scale (CPRS) (Fish, 1985). The CPRS is a 73-item clinician-rated scale that includes validated positive and negative symptom subscales (Spencer et al, 1994). We also examined responder status defined as a CGI-I of ' 1 - very much improved' or
' 2 - much improved' and at least a $20 \%$ reduction in the BPRS-C at end point of the trial.

\section{EVALUATION OF ADVERSE EFFECTS}

At each visit, subjects were systematically queried about adverse events in all major symptom areas known to be affected by antipsychotics and anticholinergics. Acute EPS were evaluated using a modification of the Simpson Angus Extrapyramidal Symptoms Scale (SAEPS) (Simpson and Angus, 1970). Tardive dyskinesia was assessed at baseline and termination using the Abnormal Involuntary Movement Scale (AIMS-NIMH) (Munetz and Benjamin, 1988). Vital signs were obtained at each visit. Body mass index (BMI) was calculated as weight in $\mathrm{kg} / \mathrm{height}$ in $\mathrm{m}^{2}$. Corrected change in BMI over time was determined by subtracting the change in 50th percentile BMI of a child of the same gender and age over the same period (calculated from the Center for Disease Control (CDC) database, 2002) from the observed BMI change. Potential effects on blood cell counts, electrolytes, renal function, liver function, energy metabolism (glucose, HgbA1c, lipid profile), prolactin, and cardiac rhythm were assessed at baseline and termination. It was not possible to consistently obtain labs at the same time of day or in a fasting state.

\section{EVALUATION OF EFFECTIVENESS}

Effectiveness was assessed by the rate and time to treatment discontinuation for any reason except moving to a setting incompatible with continued participation in the study. One subject on risperidone was excluded from this analysis for that reason. This measure is influenced by therapeutic efficacy, medication tolerability, and acceptability of treatment to patients. All participants were censored at study end point (8 weeks).

\section{STATISTICAL ANALYSIS}

Descriptive statistics were obtained on demographic, covariate, and outcome variables, using means with standard deviations for continuous variables and frequency distributions for categorical variables.

The primary analyses examined each treatment group independently, focusing on differences between the week 8 measures and the corresponding baseline measures. All comparisons used an intent to treat sample, with the last postrandomization evaluation carried forward, for all subjects who had at least one postrandomization evaluation. We considered the use of mixed models but decided that with the relatively small sample size the asymptotics were untrustworthy. The signed rank (SR) test was used for within-group comparisons of continuous measures of psychiatric symptomatology due to the small sample sizes within each group and skewness of many of these response variable's distributions. Within-group differences of the presence or absence of akathisia were analyzed using McNemar's test (Rosner, 1995). Between-group comparisons of continuous symptomatology measures used the Kruskal-Wallis nonparametric analysis of variance (ANOVA) on change scores for the same reason. Continuous 
safety variables were analyzed using ANOVA if the distributions appeared close to normal; otherwise, they were analyzed in the same manner as the continuous efficacy variables. Between-group comparisons for categorical values were analyzed using the Fischer's Exact test. All tests were two-tailed. The Bonferroni correction was used to adjust for comparison of the six continuous symptom measures within each of the three treatment groups (ie calculated $p$-values were multiplied by 6). However, no adjustments were made for multiple comparisons in the analyses of various adverse events because of the importance of being very sensitive to potential adverse events. No adjustment for multiple comparisons was made in the between-group analyses because these analyses were exploratory and not inferential. Time to event analyses used Kaplan-Meier estimates with log-rank tests to evaluate the coincidence of the survival curves in each treatment group for time to treatment response and for time to treatment discontinuation. Exploratory analyses also examined the differences between response status in different demographic groups.

\section{RESULTS}

\section{Participant Characteristics}

A total of 160 children and adolescents between the ages of 8 and 19 years, whose treating clinicians felt they required treatment for psychosis, were referred to the study between November 1997 and May 2001 as shown in Figure 1. In all, 50 had positive psychotic symptoms of at least moderate intensity and chose to enroll in the study; 19 participants were randomized to risperidone treatment, 16 to olanzapine treatment, and 15 to haloperidol treatment. The demographic and psychiatric characteristics of the sample across treatment groups are summarized in Table 1 . The mean age was 14 years 8 months $(\mathrm{SD}=32$ months, range 8 years 4 months-19 years 8 months). Of these, $60 \%$ were male, $60 \%$ were Caucasian, $32 \%$ were African-American, and $4 \%$ each were Native American and Hispanic.

Participants were severely ill with a mean $( \pm$ SD) CGI severity score of $5.6( \pm 1.1)$ and a mean $( \pm$ SD) BPRS-C total score of $51.3( \pm 12.4)$. Most $(74 \%)$ were hospitalized at enrollment with no difference in the proportion of inpatients within different treatment groups (risperidone $79 \%$, olanzapine $75 \%$, haloperidol $67 \% ; \mathrm{F}=0.77, \mathrm{df}=2$, $p=0.467)$. Most $(78 \%)$ were experiencing their first episode of psychosis. In total, 18 had never been treated with an antipsychotic and 21 had received a brief trial of an antipsychotic (typically lasting less than 2 weeks) before enrollment. Of the 11 with recurrent psychosis, four were on no antipsychotics upon entry and two had never been treated with antipsychotics. In all, 22 participants had been on a stable dose of antidepressant $(n=11)$, or mood stabilizer $(n=6)$, or both $(n=5)$ upon entry. In four subjects (olanzapine three, haloperidol one), antidepressants were started after at least 4 weeks of antipsychotic treatment due to persistent depressive symptoms. In no cases were mood stabilizers started. In two cases (one person in olanzapine group treated with both agents, one person in haloperidol group on an antidepressant), thymoleptic medications were weaned after 4 weeks of antipsychotic treatment because there were no apparent affective symptoms.

Table I Subject Characteristics

\begin{tabular}{|c|c|c|c|c|c|c|c|}
\hline \multirow[b]{2}{*}{ Characteristic } & \multirow{2}{*}{$\begin{array}{l}\text { Entire sample } \\
\qquad \begin{array}{l}N=\mathbf{5 0}\end{array}\end{array}$} & \multirow{2}{*}{$\begin{array}{c}\text { Risperidone } \\
\qquad=19\end{array}$} & \multirow{2}{*}{$\begin{array}{l}\text { Olanzapine } \\
\qquad N=16\end{array}$} & \multirow{2}{*}{$\begin{array}{l}\text { Haloperidol } \\
\qquad N=15\end{array}$} & \multicolumn{3}{|c|}{ Group differences } \\
\hline & & & & & Statistic & df & $p$ \\
\hline Male, n (\%) & $30(60)$ & $13(68)$ & $9(56)$ & 53 & $\chi^{2}=0.93$ & 2 & 0.63 \\
\hline Caucasian, n (\%) & $30(60)$ & $9(47)$ & $10(63)$ & 73 & $\chi^{2}=2.42$ & 2 & 0.30 \\
\hline Schizophrenia spectrum, n (\%) & $26(52)$ & $13(68)$ & $5(3 \mid)$ & $8(53)$ & $\chi^{2}=4.82$ & 2 & 0.09 \\
\hline Affective disorders, $n$ (\%) & $24(48)$ & $6(32)$ & II (69) & $7(47)$ & $\chi^{2}=4.82$ & 2 & 0.09 \\
\hline Age at Ist psychotic Sxs & $12.4(3.9)$ & II.4 (5.1) & $12.6(2.9)$ & I3.4 (2.9) & $F=1.20$ & 2,47 & 0.31 \\
\hline Ist psychotic episode, $n$ (\%) & $39(78)$ & $15(79)$ & $12(75)$ & $12(80)$ & $\chi^{2}=3.57$ & 2 & 0.18 \\
\hline I st hospitalization, n (\%) & $26(52)$ & $13(68)$ & $6(38)$ & $7(47)$ & $\chi^{2}=3.57$ & 2 & 0.17 \\
\hline Atypical antipsychotic, n (\%) & $28(56)$ & $12(63)$ & $7(44)$ & $8(53)$ & $\chi^{2}=1.32$ & 2 & 0.52 \\
\hline Prior antidepressant, $n$ (\%) & $25(50)$ & $9(47)$ & $8(50)$ & $8(53)$ & $\chi^{2}=0.79$ & 2 & 0.67 \\
\hline Prior mood stabilizer, $n$ (\%) & $15(30)$ & $5(26)$ & $4(25)$ & $6(40)$ & \multicolumn{2}{|c|}{ Fisher's exact } & 0.68 \\
\hline
\end{tabular}

aDiagnoses were grouped into schizophrenia spectrum, which includes schizophrenia, schizoaffective disorder, schizophreniform disorder and delusional disorder, and affective disorders, which includes major depression with psychotic features, bipolar affective disorder with psychotic features and one case of psychosis NOS with comorbid depressive disorder NOS, because of the small number of subjects in each cell otherwise. 
The treatment groups were comparable in age, age at onset, gender, ethnicity, initial BPRS-C and CPRS total scores, CPRS positive and negative symptoms subscale scores, initial CGI severity score, and prior antipsychotic exposure. Despite random assignment of patients, there was a notable difference between the groups with regard to diagnosis, though it was not statistically significant. Half of the subjects with schizophrenia spectrum disorders (13/26) were in the risperidone group, while a similar proportion of subjects with affective psychoses $(46 \%, 11 / 24)$ were in the olanzapine group $\left(\chi^{2}=4.82, \mathrm{df}=2, p<0.09\right)$.

The mean $( \pm S D)$ antipsychotic doses used at termination were risperidone $4.0( \pm 1.2) \mathrm{mg}$, olanzapine 12.3 $( \pm 3.5) \mathrm{mg}$, and haloperidol $5.0( \pm 2.0) \mathrm{mg}$. There were no statistically significant differences between the groups with regard to the use or dose of any adjunctive medications including antidepressants and mood stabilizers at baseline or termination ( $p$ ranged from 0.76 to 0.08 - Table 2). There was no difference in the intensity of psychosocial treatments received by participants in the different treatment groups as reflected by participation in in-patient or residential treatment for the majority of the trial (risperidone 58\%, olanzapine $63 \%$, and haloperidol $60 \%, \mathrm{~F}=0.027, \mathrm{df}=2$, $p=0.97)$.

\section{SYMPTOM REDUCTION}

Marked reductions in the total BPRS-C scores were observed in each of the treatment groups (Tables 3a and $\mathrm{b}$, and Figure 2). The magnitude of the reductions observed were clinically and statistically significant, with the end point BPRS-C score equal to $50 \%$ of the baseline score in the risperidone group $(\mathrm{SR}=-84.5, p=0.0018), 44 \%$ of the baseline score in the olanzapine group $(S R=-66.0$, $p=0.0018)$, and $67 \%$ of the baseline score in the haloperidol group $(\mathrm{SR}=-45.0, p=0.014)$. Similar, statistically significant reductions were observed in the positive symptoms subscale score of the CPRS, with the end point positive score equal to $52 \%$ of the baseline score in the risperidone group ( $\mathrm{SR}=-78.5, p=0.0018$ ), $37 \%$ of the baseline score in the olanzapine group ( $\mathrm{SR}=-58.5, p=0.0036$ ), and $55 \%$ of the baseline score in the haloperidol group ( $\mathrm{SR}=-48.0$, $p=0.015$ ). The negative symptoms subscale score of the CPRS was significantly reduced in the risperidone group (SR $=-70.5, p=0.005)$, but not in the olanzapine or haloperidol groups. In both the risperidone and olanzapine groups, all remaining symptom measures showed significant improvement as shown in Table 3a. However, in the haloperidol-treated group, reductions in the total CPRS, CGI-S, or CGI-I scores were not statistically significant. Over the course of the entire trial, $36 / 50 \quad(72 \%)$ of participants met criteria for positive responder status. Response rates of $74 \%$ (14/19) with risperidone, $88 \%$ (14/ $16)$ with olanzapine, and $53 \%(8 / 15)$ with haloperidol were observed (Figure 3).

Between-group comparisons of the magnitude of improvement within the psychopathology outcomes failed to detect statistical differences (Tables $3 a$ and $b$ ). However, there were differences in the mean time to response: 1.6 $( \pm 1.3)$ weeks in individuals treated with olanzapine, 2.3 $( \pm 1.8)$ weeks in those treated with risperidone, and 2.4 $( \pm 1.3)$ weeks in those treated with haloperidol (log-rank test: $\chi^{2}=6.21, \mathrm{df}=2, p<0.045$ ). Exploratory analyses (data not shown) failed to reveal any relationships between response status and diagnosis, prior antipsychotic exposure, initial severity of symptoms, or age. No differences in response rate between the different treatment groups were detected among participants who were treated exclusively with an antipsychotic (risperidone nine of 13 (69\%), olanzapine nine of $10(90 \%)$, haloperidol four of five $(80 \%), \mathrm{F}=0.68, \mathrm{df}=2, p=0.513)$, treated with concomitant antidepressant alone (risperidone one of two (50\%), olanzapine three of three (100\%), haloperidol one of four $(25 \%), \mathrm{F}=2.33, \mathrm{df}=2, p=0.178)$, treated with concomitant

Table 2 Study Medications

Analysis of between-group differences

\begin{tabular}{|c|c|c|c|c|c|}
\hline & & & & & \\
\hline & $\begin{array}{l}\text { Risperidone } \\
(N=19)\end{array}$ & $\begin{array}{l}\text { Olanzapine } \\
(N=16)\end{array}$ & $\begin{array}{l}\text { Haloperidol } \\
\quad(N=15)\end{array}$ & Statistic & $p$ \\
\hline Antipsychotic dose, mg (SD) & $3.3(1.5)$ & $12.3(4.5)$ & $5.3(1.7)$ & Not applicable & \\
\hline Amantadine, $n(\%)$ & $2(11)$ & $0(0)$ & I (7) & Fisher's exact & 0.44 \\
\hline Propranolol, n (\%) & I (5) & $2(13)$ & $0(0)$ & Fisher's exact & 0.37 \\
\hline Any mood med at end, $n(\%)$ & $6(32)$ & $8(50)$ & $10(67)$ & Fisher's exact & 0.13 \\
\hline Initial antidepressant (AD) ${ }^{\mathrm{a}}, \mathrm{n}(\%)$ & $5(26)$ & $5(31)$ & $6(40)$ & Fisher's exact & 0.70 \\
\hline AD at trial's end ${ }^{b}, n(\%)$ & $5(26)$ & $7(44)$ & $6(40)$ & Fisher's exact & 0.54 \\
\hline Initial mood stabilizer (MS) ${ }^{\mathrm{a}}, n(\%)$ & $3(16)$ & $2(13)$ & $6(40)$ & Fisher's exact & 0.36 \\
\hline MS at trial's end ${ }^{\mathrm{b}}, \mathrm{n}(\%)$ & $3(16)$ & I (6) & $6(40)$ & Fisher's exact & 0.06 \\
\hline
\end{tabular}

ancludes subjects on both AD and MS under each category.

${ }^{b}$ Accounts for subjects in which antidepressants or mood stabilizers were added or withdrawn. 
Table 3a Acute Symptom Reduction Associated with Treatment

\begin{tabular}{|c|c|c|c|c|c|c|c|c|c|c|c|c|c|c|c|c|c|}
\hline \multirow[b]{2}{*}{ Rating $^{\mathbf{a}}$} & \multicolumn{5}{|c|}{ Risperidone $(N=19)$} & \multicolumn{5}{|c|}{ Olanzapine $(N=16)$} & \multicolumn{5}{|c|}{ Haloperidol $(N=15)$} & \multicolumn{2}{|c|}{$\begin{array}{c}\text { Group } \\
\text { differences }\end{array}$} \\
\hline & Baseline & $\begin{array}{l}\text { Week } 8 \text { or } \\
\text { end point }\end{array}$ & SR & $\begin{array}{c}\text { Corrected } \\
p^{b}\end{array}$ & $\begin{array}{l}\text { Effect } \\
\text { size }\end{array}$ & Baseline & $\begin{array}{l}\text { Week } 8 \text { or } \\
\text { end point }\end{array}$ & SR & Corrected $p$ & $\begin{array}{l}\text { Effect } \\
\text { size }\end{array}$ & Baseline & $\begin{array}{l}\text { Week } 8 \text { or } \\
\text { end point }\end{array}$ & SR & Corrected $p$ & $\begin{array}{l}\text { Effect } \\
\text { size }\end{array}$ & $\mathbf{K}-\mathbf{W} \chi^{2}$ & $\mathbf{p}$ \\
\hline BPRS-C & $54 \pm 13$ & $27 \pm 20$ & -84.5 & 0.0018 & -1.44 & $50 \pm 10$ & $22 \pm 12$ & -66.0 & 0.0018 & -1.69 & $49 \pm 14$ & $33 \pm 19$ & -45.0 & 0.012 & -0.79 & 3.22 & 0.200 \\
\hline CPRS-total & $114 \pm 32$ & $60 \pm 48$ & -79.0 & 0.0018 & -1.13 & $105 \pm 25$ & $53 \pm 27$ & -51.5 & 0.0036 & -1.69 & $110 \pm 35$ & $70 \pm 39$ & -36.5 & 0.285 & -0.79 & 1.75 & 0.416 \\
\hline CPRS-positive & $27 \pm 7$ & $14 \pm 12$ & -78.5 & 0.0018 & -1.12 & $30 \pm 7$ & $11 \pm 9$ & -58.5 & 0.0036 & -1.82 & $31 \pm 6$ & $17 \pm 11$ & -48.0 & 0.015 & -1.22 & 2.76 & 0.252 \\
\hline CPRS-negative & $22 \pm 15$ & $10 \pm 8$ & -70.5 & 0.005 & -1.00 & $17 \pm 14$ & $11 \pm 9$ & -17.0 & 0.60 & -0.52 & $18 \pm 9$ & $11 \pm 10$ & -20.5 & 0.96 & -0.72 & 0.76 & 0.47 \\
\hline CGl-severity & $5.8 \pm 1.2$ & $3.8 \pm 1.3$ & -57.5 & 0.0054 & -1.22 & $5.3 \pm 1.2$ & $3.6 \pm 1.3$ & -45.5 & 0.0036 & -1.45 & $5.6 \pm 1.0$ & $4.5 \pm 1.2$ & -19.0 & 0.705 & -0.66 & 5.50 & 0.064 \\
\hline CGI-improvement & $4 \pm 0$ & $2.1 \pm 1.2$ & -86.0 & 0.0018 & -1.58 & $4 \pm 0$ & $2.0 \pm 1.1$ & -59.0 & 0.0018 & -1.83 & $4 \pm 0$ & $2.7 \pm 1.3$ & -37.5 & 0.075 & -1.03 & 3.82 & 0.15 \\
\hline
\end{tabular}

Table 3b Overall Response Associated with Treatments

\begin{tabular}{|c|c|c|c|c|}
\hline Rating $^{\mathbf{a}}$ & Risperidone $(N=19)$ & Olanzapine $(N=16)$ & Haloperidol $(N=15)$ & Group differences Fisher's exact \\
\hline Responders at week $8^{c}, n(\%)$ & $14(74)$ & $14(88)$ & $8(53)$ & 0.12 \\
\hline CGI-I of 'I', n (\%) & $6(32)$ & $5(3 \mid)$ & $2(13)$ & 0.44 \\
\hline
\end{tabular}

${ }^{a}$ All values are $L O C F$ to week 8 . All numeric values are shown as the mean $\pm S D$.

the $p$-values within each treatment group have been corrected for multiple comparisons within the group by multiplying by 6 .

'Responders were defined a priori as having a CGI of 'I' or ' 2 ' and a $20 \%$ or greater reduction in the BPRS-C total score. 


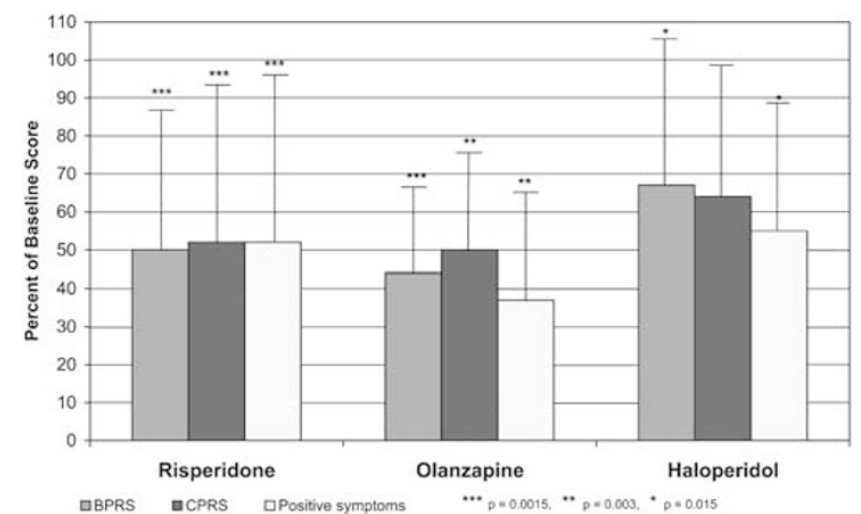

Figure 2 Symptom reduction.

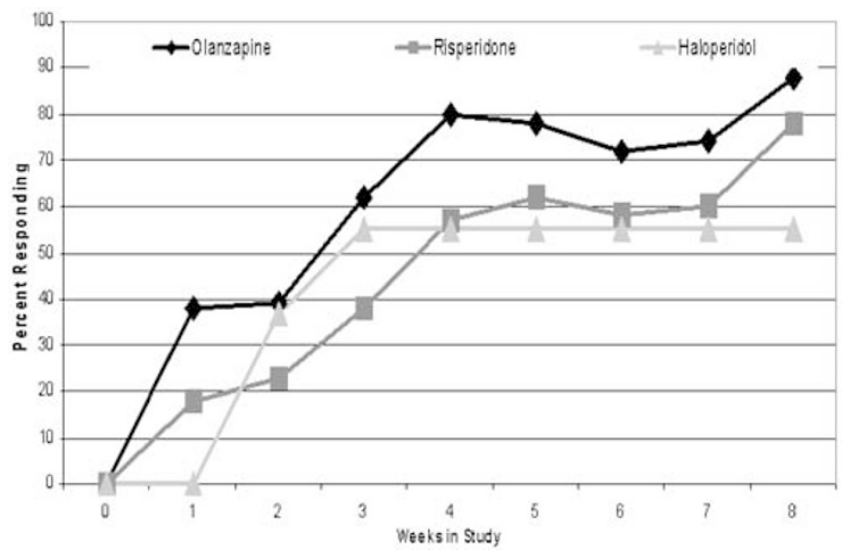

Figure 3 Response to treatment.

mood stabilizer alone (risperidone one of one (100\%), olanzapine one of one $(100 \%)$, haloperidol two of four $(50 \%), \mathrm{F}=0.550, \mathrm{df}=2, p=0.65)$, treated concomitantly with both an antidepressant and mood stabilizer (risperidone three of three $(100 \%)$, olanzapine one of two $(50 \%)$, haloperidol one of two (50\%), $\mathrm{F}=0.857, \mathrm{df}=2, p=0.49$ ), or treated with any concomitant mood medication initially (risperidone five of six (83\%), olanzapine five of six (83\%), haloperidol four of $10(40 \%), \mathrm{F}=2.39, \mathrm{df}=2, p=0.118)$.

\section{SAFETY MEASURES}

Side effects were frequently observed in this pediatric sample. More than half of the subjects treated with either atypical medication had evidence of mild to moderate Parkinsonian symptoms and two of the 19 subjects treated with risperidone had severe EPS (data not shown). Further, a large proportion of those in each treatment group required low-dose anticholinergics to control their EPS (haloperidol-67\%, olanzapine - 56\%, risperidone - 53\%). In most subjects (23 of 30 ), anticholinergics were continued throughout the remainder of the trial. The final benztropine dose was not different between treatment groups (haloperidol $0.7( \pm 1.0) \mathrm{mg} /$ day; risperidone $0.9( \pm 1.1) \mathrm{mg} /$ day; olanzapine $0.8( \pm 1.1) \mathrm{mg} / \mathrm{day},(\mathrm{SR}, \mathrm{df}=2,47 ; \mathrm{F}=0.084$, $p<0.96)$. Two individuals with acute and severe dystonic reactions in the haloperidol group were withdrawn from the study prior to starting anticholinergics. Between-group comparisons of maximal EPS demonstrated more frequent and severe symptoms in the haloperidol group (SR, $\mathrm{df}=2,47 ; \mathrm{F}=3.48, p<0.04$ overall; $p<0.04$ compared to risperidone and $p<0.02$ compared to olanzapine). Further, the mean Simpson Angus score changed significantly between baseline and end point only in the haloperidol group (Table 4a). Four subjects reported akathisia at end point; two were treated with haloperidol and two with olanzapine.

Significant weight gain was observed in all treatment groups (risperidone: $4.9( \pm 3.6) \mathrm{kg} / 10.9( \pm 7.9) \mathrm{LB}$; olanzapine: $7.1( \pm 4.1) \mathrm{kg} / 15.7 \quad( \pm 9.2) \mathrm{LB}$; haloperidol 3.5 $( \pm 3.7) \mathrm{kg} / 7.8( \pm 8.2) \mathrm{LB})$. The weight gain was disproportionate to the increases in participant height with BMI increases exceeding the developmentally expected BMI change for each subject (calculated from the CDC growth curves, $C D C, 2002$ ). The pattern of BMI changes within the different treatment groups was maintained (risperidone: 1.4 $( \pm 1.2) \mathrm{kg} / \mathrm{m}^{2}$; olanzapine: $2.3( \pm 1.2) \mathrm{kg} / \mathrm{m}^{2}$; haloperidol: $\left.1.1( \pm 1.2) \mathrm{kg} / \mathrm{m}^{2}\right)$. These between-group differences were statistically significant for both total weight gain and increase in BMI (Kruskal-Wallis test, $\chi^{2}=6.51, \mathrm{df}=2,47$; $p<0.0386$ for weight and $\chi^{2}=6.77, \mathrm{df}=2,47 ; p<0.0339$ for $\mathrm{BMI})$. Participants treated with haloperidol gained weight at the slowest rate $(0.54( \pm 0.59) \mathrm{kg} /$ week $)$, while those treated with the atypicals gained more quickly (RIS 0.77 $( \pm 0.50) \mathrm{kg} /$ week; OLA $0.99( \pm 0.50) \mathrm{kg} /$ week $)$. However, these differences were not statistically significant $\left(\chi^{2}=3.54\right.$, $\mathrm{df}=2,47 ; p<0.170)$.

The effects of each treatment on various safety parameters are summarized in Table 4a. There were three parameters, which changed significantly between baseline and end point. In the haloperidol-treated group, there was a small increase in the QTc from $394( \pm 18)$ to 402 $( \pm 16) \mathrm{ms}$, SR 18.5, $p=0.031$. Similar increases were not observed in the atypical antipsychotic-treated groups. In the risperidone-treated group, increases were observed in two liver function tests: aspartate aminotransferase (AST) increased from $21.9( \pm 5.4) \mathrm{U} / \mathrm{l}$ to $28.1( \pm 10.8) \mathrm{U} / \mathrm{l}, \mathrm{SR}$ $35, p=0.046$ and alanine aminotransferase (ALT) increased from $20.9( \pm 11.9) \mathrm{U} / \mathrm{l}$ to $32.9( \pm 23.8) \mathrm{U} / \mathrm{l}, \mathrm{SR} 39.5$, $p=0.0104$. There was also a trend toward an increase in the random glucose within the olanzapine-treated group from $87.2( \pm 10.8) \mathrm{mg} / \mathrm{dl}$ to $97.2( \pm 14.4) \mathrm{mg} / \mathrm{dl}$, SR 18.5 , $p=0.0645$. However, the clinical significance of these increases is unclear. Between-group comparisons of these parameters demonstrated significant differences between groups only with regard to AST elevations (Kruskal-Wallis $\chi^{2}=6.62, p=0.036$ ) and random glucose elevations (Kruskal-Wallis $\left.\chi^{2}=9.74, p=0.008\right)$. Although the mean end point prolactin level was greater in the risperidone-treated group $(37.2 \pm 19.8 \mathrm{ng} / \mathrm{ml})$ than the other groups, (olanzapine $30.0 \pm 12.9 \mathrm{ng} / \mathrm{ml}$ and haloperidol $32.2 \pm 29.0)$ this difference was not significant. Further, the reports of side effects believed to be related to prolactin elevations were similar between groups (menstrual irregularities (risperidone - 1 , olanzapine - 0 , haloperidol-2), gynecomastia and small, painful masses of breast tissue (risperidone -2 , olanzapine -2 , haloperidol-2), and galactorrhea (risperidone-1, olanzapine-1, haloperidol-0) and were not clearly related to prolactin levels. 
Table 4a Acute Objective Side Effects of Treatment

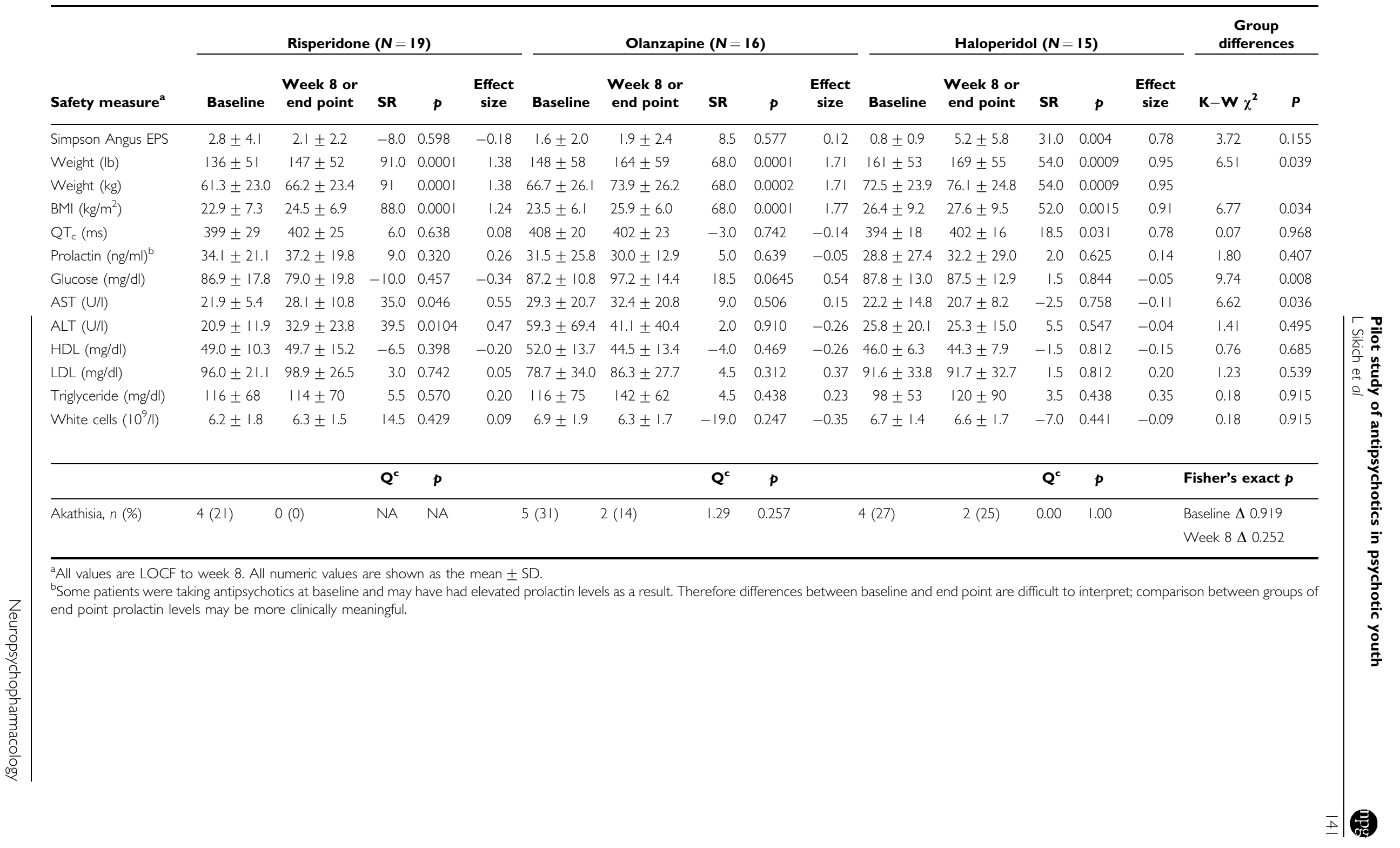


Table 4b Elicited Subjective Side Effects of Treatment

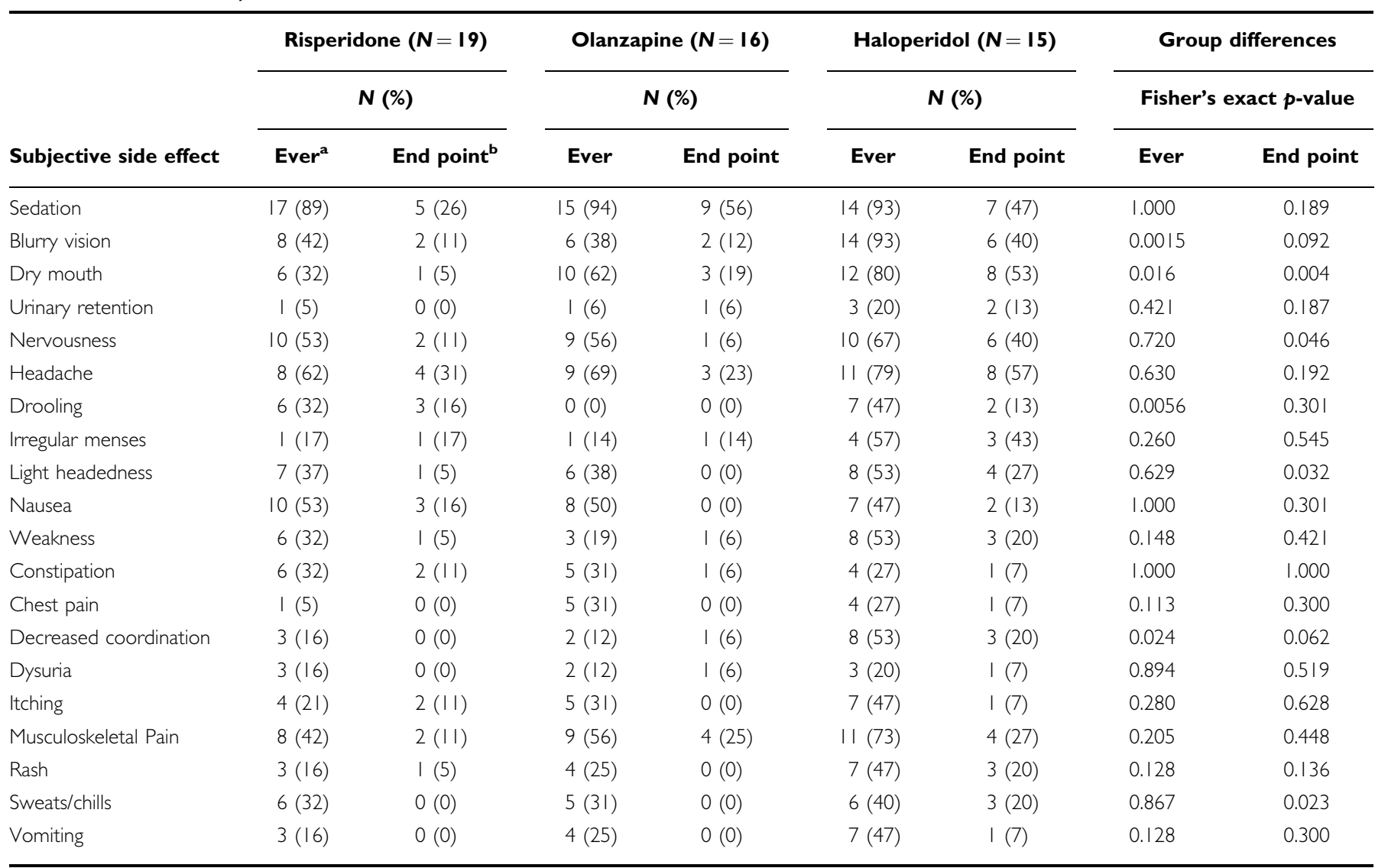

${ }^{a}$ Reported at any point during the subject's participation in the study.

${ }^{\mathrm{b}}$ Reported during the last visit.

Side effects that were elicited at any time during the trial in $20 \%$ or more of all subjects are listed in Table $4 \mathrm{~b}$. The most frequently reported side effects were sedation (92\%), nervousness $(58 \%)$, dry mouth (56\%), blurry vision $(56 \%)$, headache (56\%), musculoskeletal pain (56\%), nausea $(54 \%)$, and light headedness (42\%). Most of these resolved over time as shown in Table $4 \mathrm{~b}$. However, approximately half of individuals treated with olanzapine (56\%) and haloperidol (47\%) continued to complain of sedation, while only a quarter of those treated with risperidone did. Headache and blurry vision were also fairly persistent, particularly in the haloperidol group. There were a limited number of reported side effects that occurred with significantly different rates in different treatment groups. The olanzapine-treated group did not experience drooling at any point in contrast to the other groups. The haloperidol-treated group more frequently reported dry mouth and decreased coordination during the trial, and lightheadedness and sweats/chills at termination.

\section{EFFECTIVENESS}

This 8-week acute treatment study was completed by 32 of the 50 subjects (64\%). The ability of subjects to sustain treatment varied considerably between the study medications. Two of $16(13 \%)$ participants withdrew from the olanzapine group, while nine of $19(47 \%)$ participants withdrew from the risperidone group, and seven of 15 (47\%) participants withdrew from the haloperidol group. One of the subjects from the risperidone group withdrew for administrative rather than clinical reasons. This betweengroup difference approached statistical significance (Fisher's exact $p=0.058$ ). This was reflected in a somewhat longer duration of treatment for those patients receiving olanzapine $(7.4( \pm 1.9)$ weeks), than those receiving risperidone (6.3 $( \pm 2.3)$ weeks) and haloperidol (5.7 ( \pm 2.7 ) weeks). However, these differences were not statistically significant (log-rank test: $\chi^{2}=4.68, \mathrm{df}=2$, $p<0.096)$.

The time course and precipitants of treatment discontinuation also varied between groups (Table 5). All but one of the participants who discontinued treatment with haloperidol did so within the first 3 weeks. Termination was often precipitated by EPS $(n=5)$. In two of these five cases, the participants had not received adjunctive anticholinergics; in one case, EPS was coupled with inability to tolerate blurry vision caused by benztropine. In contrast, participants who withdrew from atypical antipsychotic treatment did so throughout the 8-week trial. Mania precipitated discontinuation in one patient treated with risperidone and thrombocytopenia precipitated discontinuation in a patient treated with haloperidol. Insufficient response precipitated both of the withdrawals from the olanzapine group. 


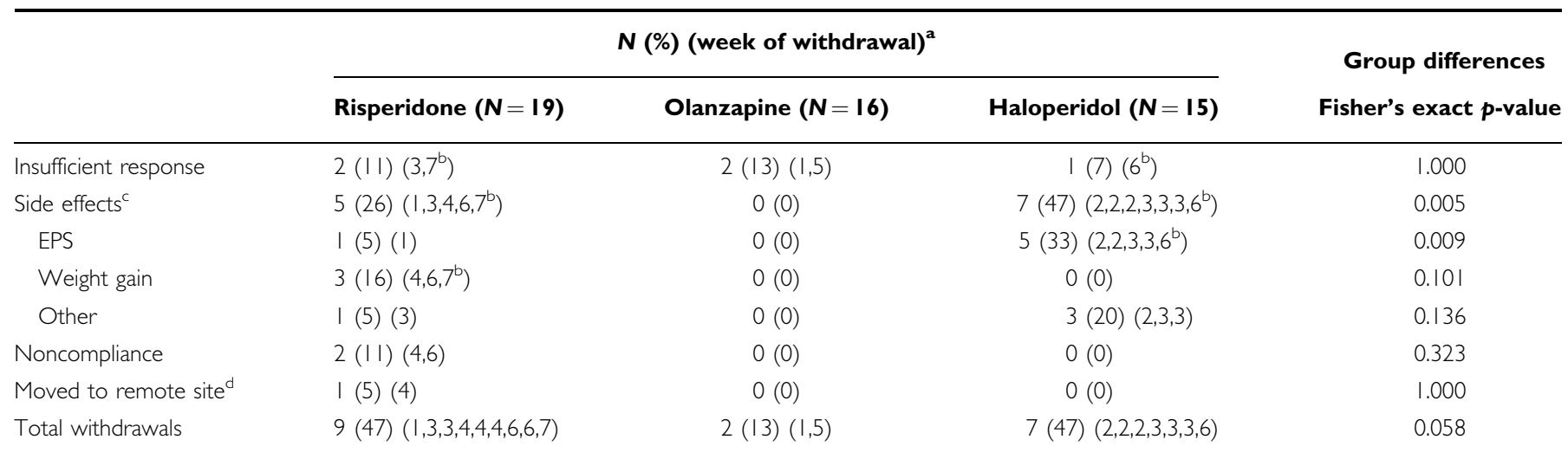

aThe week that each subject withdrew for that reason is shown in brackets in order to provide a sense of the time course of such events.

${ }^{b}$ Subject withdrew as a result of both insufficient response and intolerable side effects (weight gain for the risperidone-treated subject and extrapyramidal symptoms for the haloperidol-treated subject).

'The withdrawals due to any side effect are shown first, with those due to specific side effects listed below. In the haloperidol, group one subject withdrew as a result of both EPS and anticholinergic side effects.

${ }^{d}$ If the subject who moved to a remote site is excluded from this analysis, $8 / 18(44 \%)$ in the risperidone group withdrew.

\section{DISCUSSION}

Although double-blind trials of atypicals have been conducted in youth with bipolar disorder and with behavioral problems, this paper presents data from the first randomized, double-blind trial of first-line atypical antipsychotics in youth presenting for treatment of psychotic symptoms. The results provide preliminary evidence that these atypical antipsychotics - risperidone and olanzapine - reduce psychotic symptoms to at least as great an extent as the conventional antipsychotic haloperidol. Further, there is a trend toward greater improvement on all of the measures assessed, including total psychopathology scores on the BPRS-C and CPRS, the positive symptom subscale of the CPRS, CGI-severity and -improvement scores, and overall response rate that is not statistically significant.

This study also provides evidence that moderate doses of risperidone and olanzapine are associated with side effects in youth that are more prevalent and severe than those reported in psychotic adults. The majority of participants treated with atypicals experienced at least mild to moderate EPS. These findings are consistent with earlier work suggesting that youth are particularly sensitive to EPS (Keepers et al, 1983). Although we identified no cases of tardive dyskinesia in this study, the acute EPS observed with the atypical agents in this study suggests the need to monitor for tardive dyskinesia. Almost all youth experienced sedation during the course of treatment, despite relatively low-dose treatment and slow titration. However, the sedation generally dissipated over time, particularly in the risperidone-treated group. Most importantly, most of the youth in this study gained considerable weight. These data raise concerns that children treated with these antipsychotics may be at increased risk for chronic health problems associated with obesity such as diabetes, hypertension, cardiovascular disease, and osteoarthritis. Additional research is needed to characterize the long-term course of antipsychotic-associated weight gain in youth, any associated metabolic changes and the relationship between resulting health problems and patient characteristics such as age, sex, diagnosis, treatment response, initial body weight, family history, and genetic vulnerability factors. It will also be important to explore possible adjunctive behavioral and pharmacologic strategies for limiting antipsychotic-associated weight gain in youth. Studies are also needed to elucidate whether different or additional mechanisms are involved in antipsychotic-associated weight gain in youth than in adults.

\section{LIMITATIONS}

This pilot study has a number of limitations, including limited sample size, differences in the diagnoses of participants in the different treatment groups, use of concomitant medications, and variations in age and pubertal status. The small sample size limits the study's ability to detect moderate to small differences between treatments that may be clinically significant. The limited sample size also makes it difficult to do any sort of subgroup analyses, thus increasing the likelihood that important differences between groups will not be recognized.

In addition, the study is limited by the differences in the diagnostic composition of the treatment groups, particularly the olanzapine and risperidone groups. It is not possible to assess fully the extent to which observed differences in response between treatment groups are related to subtle differences in the symptom profile and treatment responsiveness of affective psychoses and schizophrenia spectrum illnesses. For instance, if schizophrenia spectrum illnesses are associated with greater treatment resistance than affective psychoses, the response rate of the risperidone group might be artifactually reduced. Similarly, the finding of significant changes in negative symptoms with risperidone treatment, but not olanzapine or haloperidol treatment, could be related to higher baseline negative 
symptoms in the risperidone group, which, in turn, might be related to the tendency for individuals with schizophrenia to have slightly more severe negative symptoms than participants with other diagnoses. Finally, diagnostic differences may influence the use of or response to concomitant medications.

Another significant limitation of this pilot study is the use of a variety of adjunctive antidepressants and/or mood stabilizers in 22 of 50 subjects initially and 24 of 50 after 4 weeks. Although there are not statistical differences in the use of particular classes of adjunctive medications between the treatment groups, it is possible that observed differences in efficacy and/or side effects between treatment groups are related to one or more of these adjunctive medications rather than the antipsychotic itself. However, no obvious relationship was detected between response rate and adjunctive use of thymoleptic medications.

Finally, interpretation of metabolic side effects, particularly glucose levels, is considerably limited by the random timing of samples throughout the day without clear relationship to meals. Interpretation of these measures, as well as prolactin levels, is further complicated by the broad range of ages and pubertal development within the sample.

\section{CLINICAL IMPLICATIONS}

In summary, this study provides initial controlled data that risperidone and olanzapine are effective in reducing psychotic symptoms in children and adolescents with a variety of diagnoses. These two atypical agents have lower risk for acute, severe EPS than the traditional agent haloperidol. However, EPS is identified at much higher rates than observed in comparable adult populations. Weight gain is found in all treatment groups and remains an important concern. Additional, larger studies, which stratify for diagnosis and restrict the acute use of adjunctive mood medications, are needed to fully evaluate potential differences in the efficacy and tolerability of the atypical agents. In addition, comparisons with other atypicals appear indicated given the propensity for problematic side effects in both the risperidone and olanzapine groups. Finally, the high discontinuation rate highlights the importance of developing antipsychotic medications that are more effective and better-tolerated in the pediatric population.

\section{ACKNOWLEDGEMENTS}

This study was supported in part by NIMH grant (MH53551), the UNC-Mental Health and Neuroscience Clinical Research Center ( $\mathrm{MH}$ MH33127), NIH grant (RR00046) from the General Clinical Research Centers program, Janssen Pharmaceutica, Eli Lilly Company (donated olanzapine), and the Foundation of Hope (Raleigh, North Carolina). The authors thank A Malekpour, K Williamson, T Brooks, R White, J Hughes, D Ekstrom, C Jackson, S Brown, and K Kennedy for their assistance. Most of all the authors are deeply grateful to the patients who participated and their families. Data from this study were presented at the 2001 American College of Neuropsychopharmacology Meeting, San Juan, Puerto Rico, and at the
2002 Society of Biological Psychiatry Convention, Philadelphia, PA.

\section{REFERENCES}

Aman MG, De Smedt G, Derivan A, Lyons B, Findling RL (2002). Risperidone Disruptive Behavior Study Group. Double-blind, placebo-controlled study of risperidone for the treatment of disruptive behaviors in children with subaverage intelligence. Am J Psychiatry 159: 1337-1346.

Buitelaar JK, van der Gaag RJ, Cohen-Kettenis P, Melman CT (2001). A randomized controlled trial of risperidone in the treatment of aggression in hospitalized adolescents with subaverage cognitive abilities. J Clin Psychiatry 62: 239-248.

Buitelaar JK, Willemsen-Swinkels SH (2000). Medication treatment in subjects with autistic spectrum disorders. Eur Child Adolesc Psychiatry 9(Suppl 1): I85-197.

CDC (2002). http://www.cdc.gov/nccdphp/dnpa/bmi/bmi-forage.htm, September 2002, CDC BMI for age tables.

Chambers W, Puig-Antich J, Hirsch M, Paez P, Ambrosini J, Tabrizi MA (1985). The assessment of affective disorders in children and adolescents by semi-structured interview: testretest reliability of the Schedule for Affective Disorders and Schizophrenia for school-age children, present episode version. Arch Gen Psychiatry 42: 696-702.

DelBello MP, Schwiers ML, Rosenberg HL, Strakowski SM (2002). A double-blind, randomized, placebo-controlled study of quetiapine as adjunctive treatment for adolescent mania. J Am Acad Child Adolesc Psychiatry 41: 1216-1223.

Emslie GJ, Rush AJ, Weinberg WA, Kowatch RA, Hughes CW, Carmody $\mathrm{T}$ et al (1997). A double-blind, randomized placebocontrolled trial of fluoxetine in depressed children and adolescents. Arch Gen Psychiatry 54: 1031-1037.

Findling RL, McNamara NK, Branicky LA, Schluchter MD, Lemon E, Blumer JL (2000). A double-blind pilot study of risperidone in the treatment of conduct disorder. J Am Acad Child Adolesc Psychiatry 3: 509-516.

First MB, Gibbon M, Spitzer RL, Williams J (1997). Structured Clinical Interview for DSM-IV Axis I Disorders (SCID-I), Clinician Version. American Psychiatric Press, Inc.: Washington, DC.

Fish B (1985). Children's Psychiatric Rating Scale. Psychopharmacol Bull 21: 753-770.

Guy W (1976). ECDEU Assessment Manual for Psychopharmacology Revised DHEW Pub. No. (ADM) 76-338. National Institute of Mental Health: Rockville, MD.

Henderson DC (2002). Diabetes mellitus and other metabolic disturbances induced by atypical antipsychotic agents. Curr Diabet Reports 2: 135-140.

Hughes CW, Rintelmann J, Emslie GJ, Lopez M, MacCabe N (2001). A revised anchored version of the BPRS-C for childhood psychiatric disorders. J Child Adol Psychopharmacol 11: 77-93.

Kafantaris V, Coletti DJ, Dicker R, Padula G, Kane JM (2001a). Adjunctive antipsychotic treatment of adolescents with bipolar psychosis. J Am Acad Child Adolesc Psychiatry 40: 1448-1456.

Kafantaris V, Dicker R, Coletti DJ, Kane JM (2001b). Adjunctive antipsychotic treatmen is necessary for adolescents with psychotic mania. J Child Adolesc Psychopharmacol 11: 409-413. Keepers GA, Clappison VJ, Casey DE (1983). Initial anticholinergic prophylaxis for acute neuroleptic induced extrapyramidal syndromes. Arch Gen Psychiatry 40: 1113-1117.

Kelly DL, Conley RR, Love RC, Horn DS, Ushchak CM (1998). Weight gain in adolescents treated with risperidone and conventional antipsychotics over six months. J Child Adolesc Psychopharmacol 8: 151-159. 
Kumra S (2000). The diagnosis and treatment of children and adolescents with schizophrenia: My mind is playing tricks on me. Child Adolesc Psychiatric Clin North Am 9: 183-199.

Kumra S, Frazier JA, Jacobsen L, McKenna K, Gordon CT, Lenane $M$ et al (1996). Childhood-onset schizophrenia: a double-blind clozapine-haloperidol comparison. Arch Gen Psychiatry 53: 1090-1097.

Lieberman JA (1996). Atypical antipsychotic drugs as a first line treatment of schizophrenia: a rationale and hypothesis. J Clin Psychiatry 57(s11): 68-71.

Malone RP, Cater J, Sheikh RM, Choudhury MS, Delaney MA (2001). Olanzapine versus haloperidol in children with autistic disorder: an open pilot study. J Am Acad Child Adolesc Psychiatry 40: 887-894.

Malone RP, Sheikh R, Zito JM (1999). Novel antipsychotic medications in the treatment of children and adolescents. Psychiatr Serv 50: 171-174.

Martin A, Landau J, Leebens P, Ulizio K, Cicchetti D, Scahill L et al (2000). Risperidone-associated weight gain in children and adolescents: a retrospective chart review. J Child Adolesc Psychopharmacol 10: 259-268.

McClellan J, McCurry C, Speltz ML, Jones K (2002). Symptom factors in early-onset psychotic disorders. J Am Acad Child Adolesc Psychiatry 41: 791-798.

McCracken JT, McGough J, Shah B, Cronin P, Hong D, Research Units on Pediatric Psychopharmacology Autism Network et al (2002). Risperidone in children with autism and serious behavioral problems. $N$ Engl J Med 347: 314-321.

McKenna K, Gordon CT, Lenane M, Kaysen D, Fahey K, Rapoport JL (1994). Looking for childhood onset schizophrenia: the first 71 cases screened. J Am Acad Child Adolesc Psychiatry 33: 636-644.

Munetz MR, Benjamin S (1988). How to examine patients using the Abnormal Involuntary Movement Scale. Hosp Community Psychiatry 39: 1172-1177.

Newcomer JW, Haupt DW, Fucetola R, Melson AK, Schweiger JA, Cooper BP et al (2002). Abnormalities in glucose regulation during antipsychotic treatment of schizophrenia. Arch Gen Psychiatry 59: 337-345.

Overall JE, Gorham DR (1962). The brief psychiatric rating scale. Psychol Rep 10: 799-812.

Overall JE, Pfefferbaum B (1982). The Brief Psychiatric Rating Scale for children. Psychopharmacol Bull 18: 10-16.

PMATSLS2 (2002). http://www.IMShealth.com, Retail \& Provider Perspective Audit (PMATSLS2).
Pool D, Bloom W, Mielke DH, Roniger Jr JJ, Gallant DM (1976). A controlled evaluation of loxitane in seventy-five adolescent schizophrenic patients. Curr Ther Res Clin Exp 19: 99-104.

Ratzoni G, Gothelf D, Brand-Gothelf A, Reidman J, Kikinzon L, Gal $\mathrm{G}$ et al (2002). Weight gain associated with olanzapine and risperidone in adolescent patients: a comparative prospective study. J Am Acad Child Adolesc Psychiatry 41: 337-343.

Remschmidt H, Hennighausen K, Clement HW, Heiser P, Schulz E (2000). Atypical neuroleptics in child and adolescent psychiatry. Eur Child Adolesc Psychiatry 9(Suppl 1): 9-19.

Rosner B (1995). Hypothesis testing: categorical data. In: Wadswort Publishing Company (eds). Fundamentals of Biostatistics. Belmont: CA.

Selva KA, Scott SM (2001). Diabetic ketoacidosis associated with olanzapine in an adolescent patient. J Pediatr 138: 936-938.

Sernyak MJ, Leslie DL, Alarcon RD, Losonczy MF, Rosenheck R (2002). Association of diabetes mellitus with use of atypical neuroleptics in the treatment of schizophrenia. Am J Psychiatry 159: $561-566$.

Simpson GM, Angus JW (1970). A rating scale for extrapyramidal side effects. Acta Psychiatr Scand 212(Suppl): 11-19.

Snyder R, Turgay A, Aman M, Binder C, Fisman S, Carroll A (2002). The Risperidone Conduct Study Group. Effects of risperidone on conduct and disruptive behavior disorders in children with subaverage IQs. J Am Acad Child Adolesc Psychiatry 41: 1026-1036.

Spencer EK, Alpert M, Pouget ER (1994). Scales for the assessment of neuroleptic response in schizophrenic children: specific measures derived from the CPRS. Psychopharmacol Bull 30: 199-202.

Spencer EK, Campbell M (1994). Children with schizophrenia: diagnosis, phenomenology, and pharmacotherapy. Schizophr Bull 20: 713-725.

Toren P, Laor N, Weizman A (1998). Use of atypical neuroleptics in child and adolescent psychiatry. J Clin Psychiatry 59: 644-656.

Werry JS, McClellan J, Chard L (1991). Early-onset schizophrenia, bipolar and schizoaffective disorders: a clinical follow-up study. J Am Acad Child Adolesc Psychiatry 30: 457-465.

Wirshing DA, Spellberg BJ, Erhart SM, Marder SR, Wirshing WC (1998). Novel antipsychotics and new onset diabetes. Biol Psychiatry 44: 778-783.

Wudarsky M, Nicolson R, Hamburger SD, Spechler L, Gochman P, Bedwell J et al (1999). Elevated prolactin in pediatric patients on typical and atypical antipsychotics. J Child Adolesc Psychopharmacol 9: 239-245. 